\section{Oberrheinische Arbeitsgemeinschaft für Onkologie (OAO/GTOR/AOR)}

Oberrheinische Arbeitsgemeinschaft für Onkologie

Onkologisches Zentrum, III. Med. Klinik

Universitätsklinikum

Theodor-Kutzer-Ufer, D-68167 Mannheim

Tel. +49 621 383-2854 o. -3258, Fax -3833

E.mail petra.mura@med3.ma.uni-heidelberg.de

\section{Jean-Paul Obrecht Award 2004}

Während der 20. Jahrestagung der OAO in Colmar vom 16. bis 17. Januar 2004 wurde der Jean-Paul Obrecht Award zum fünften Mal verliehen. Ausgezeichnet werden ausschließlich Wissenschaftler unter 40 Jahren. Die Übergabe des Preises erfolgte eigens durch Herrn Prof. Dr. med. Jean-Paul Obrecht, Arlesheim, dem früheren Leiter der Abteilung Onkologie am Kantonspital (Universitätskliniken) Basel und Begründer der Gesellschaft. Den Preis erhielt in diesem Jahr

Herr Dr. med. Christoph Mamot, Basel.

Der am 28. Januar 1971 geborene Preisträger studierte von 1990 bis 1997 Medizin an den Universitäten Bochum und Freiburg. Er promovierte 1997 am Institut für Pharmakologie der Universität Freiburg. Seine klinische Ausbildung zum Internisten absolvierte er von 1997 bis 2001 in Liestal/Schweiz, Durban/Südafrika, Lörrach und Bad Krozingen/Deutschland sowie in Basel/Schweiz, dort war er ab 1998 wissenschaftlicher Mitarbeiter der Abteilung Onkologie (Leiter: Prof. Dr. med. R. Herrmann) am Kantonsspital. Von 2001 bis 2003 hatte er ein Postdoctoral Fellowship an der Abteilung Hämatologie/ Onkologie der Universität Californien San Francisco/USA inne, seitdem ist er wieder in Basel tätig. Bis heute legt er sieben Originalarbeiten vor, für seine Arbeiten errang er bereits drei Preise.

Der OAO Award 2004 wurde ihm für die Präsentation des folgenden Beitrages zuerkannt:

Mamot C, Rochlitz C, Herrmann R, Drummond DC, Hong K, Kirpotin DB, Park JW:

EGFR-Targeted Drug Delivery via Immunoliposomes. Onkologie 2003;26:600 (Abstract).

Kurzfassung:

Die unspezifische Toxizität der meisten Zytostatika ist ein unverändert limitierendes Problem in der Onkologie. Transportsysteme wie Liposomen können die Pharmakokinetik der zu transportierenden Wirkstoffe verändern und gegebenenfalls verbessern. Gegenstand unserer Forschung ist es, Liposomen durch Kopplung von rezeptor-spezifischen Antikörpern an deren Oberfläche zu rezeptor- bzw. tumorspezifischen Transportvehikeln $\mathrm{zu}$ verwandeln (Immunoliposomen, ILs). Wir haben ILs entwickelt, die gegen den EGF-Rezeptor gerichtet sind, um Wirkstoffe wie Zytostatika zielgerichteter zu EGFR überexprimierenden Tumorzellen zu transportieren. Gerade der EGF-Rezeptor scheint geeignet zu sein, da er auf Tumoren häufig überexprimiert wird und nach Bindung eines Liganden an den Rezeptor eine Internalisierung des Komplexes ausgelöst wird.

Es gelang die Entwicklung von Immunoliposomen, welche gegen den EGF- und auch EGFvIII-Rezeptor gerichtet sind. Hierzu wurden Antikörperfragmente (Fab') des monoklonalen Antikörpers C225 (Cetuximab, Erbotux ${ }^{\mathrm{TM}}$ ) bzw. sogenannte single-chain Antikörper (University of California San Francisco) benutzt. Die Antikörperfragmente werden kovalent an die Liposomen gebunden (20-30 pro Liposom).

Dabei konnte gezeigt werden, dass anti-EGFR ILs einen spezifischen Transport verschiedenster Diagnostika und Therapeutika zu EGFR-überexprimierenden Zellen ermöglichen. Quantifizierungsversuche in vitro haben ergeben, dass bis zu 13000 ILs je Tumorzelle internalisiert werden, während in nicht EGFR-überexprimierenden Tumorzellen weniger als 300 Ils pro Zelle unspezifisch aufgenommen werden. Da in jedem einzelnen Liposom meist mehr als 10000 Wirkstoffmoleküle transportiert werden können, zeigten die mit verschiedenen Wirkstoffen wie Doxorubicin, Vinorelbine oder Methotrexat beladenen anti-EGFR ILs eine verbesserte Wirksamkeit in EGFR-exprimierenden Tumorzellen. So konnte die IC $50 \mathrm{im}$ Vergleich zum entsprechenden liposomalen Wirkstoff um den Faktor 20-40 reduziert, d.h. die Wirksamkeit um diesen Faktor gesteigert werden. Hingegen zeigten anti-EGFR ILs die gleiche limitierte Wirksamkeit wie unspezifische Liposomen in EGFR negativen Zellen.

Diese Daten konnten in Tiermodellen an einer Reihe EGFRüberexprimierender Tumoren bestätigt werden. Dabei sind mit Doxorubicin oder Epirubicin beladene anti-EGFR ILs sämtlichen Kontrollen gegenüber überlegen. Somit können anti-EGFR Immunoliposomen Zytostatika effizient und spezifisch zu EGFR-überexprimierenden Tumoren transportieren, die Wirksamkeit von Zytostatika kann erhöht und deren Toxizität vermindert werden.

Prof. Dr. med. W. Queißer, Mannheim Vizepräsident der OAO/GTOR/AOR 